\title{
Electronic States in Three Dimensional Quantum Dot/Wetting Layer Structures
}

\author{
Marta Markiewicz and Heinrich Voss \\ Institute of Numerical Simulation \\ Hamburg University of Technology \\ D-21071 Hamburg, Germany \\ \{m.markiewicz, voss\}@tu-harburg.de
}

\begin{abstract}
Although self-assembled quantum dots are grown on wetting layers, most simulations exclude the wetting layer. The neglected effects on the electronic structure of a pyramidal InAs quantum dot embedded in a GaAs matrix are investigated based on the effective one electronic band Hamiltonian, the energy and position dependent electron effective mass approximation, and a finite height hard-wall 3D confinement potential. By comparing quantum dots with wetting layers and a dot without a wetting layer, we find that the presence of a wetting layer may effect the electronic structure essentially.
\end{abstract}

\section{Introduction}

Semiconductor nanostructures have attracted tremendous attention in the past few years because of their unique physical properties and their potential for applications in micro- and optoelectronic devices. In such nanostructures, the free carriers are confined to a small region of space by potential barriers, and if the size of this region is less than the electron wavelength, the electronic states become quantized at discrete energy levels. The ultimate limit of low dimensional structures is the quantum dot, in which the carriers are confined in all three directions.

Quantum dots can be produced today by the Stranski-Krastanov process which uses the relief of the elastic energy when two materials with a large lattice mismatch form an epitaxial structure. The deposited layer initially grows as a thin two dimensional (2D) wetting layer. As the deposited layer exceeds a critical thickness, the growth mode switches from 2D to 3D leading to the formation of a self-assembled quantum dot on top of the wetting layer.

Most simulations neglect the effect of wetting layers on the electronic structure of self-assembled quantum dots (cf. $[5,6,8-11,19,20]$ and the literature given therein). In this paper we report on numerical simulations investigating the effect of a wetting layer on the electronic structure of a pyramidal InAs quantum dot embedded in a GaAs matrix. We consider the one-band envelope-function formalism for electrons and holes assuming non-parabolicity for the electron's dispersion relation and an electron effective mass depending on the position and 
the energy level. Then the discretization of the Schrödinger equation results in a sparse eigenvalue problem depending nonlinearly on the eigenparameter. Similar experiments are contained in [12-15] where the authors assumed an axially symmetric quantum dot and an electron effective mass which does not depend on the energy level. These assumptions lead to linear eigenvalue problems of much smaller dimension.

Our paper is organized as follows. In Section 2 we state the rational eigenvalue problem which models the electronic behavior of a quantum dot (possibly including a wetting layer) assuming a position and energy dependent quasiparticle effective mass approximation. Discretization by a Galerkin method yields a sparse rational matrix eigenvalue problem which allows a minmax characterization of its eigenvalues. Section 3 describes the iterative projection methods introduced already in [20], and discusses the solution of the projected rational eigenproblems by safeguarded iteration. Numerical results are given in Section 4 demonstrating that the effect of the wetting layer on the electronic structure of a quantum dot is essential.

\section{Position dependent effective mass model}

We consider the problem to compute relevant energy states and corresponding wave functions of a three dimensional semiconductor quantum dot with or without a wetting layer. Let $\Omega_{q} \subset \mathbb{R}^{3}$ be a domain occupied by the quantum dot with the possible inclusion of a wetting layer, which is embedded in a bounded matrix $\Omega_{m}$ of different material. A typical example is an InAs pyramidal quantum dot grown on a wetting layer, which is embedded in a cuboid GaAs matrix (cf. Fig. $1)$.

We consider the one-band envelope-function formalism for electrons and holes in which the effective Hamiltonian is given by

$$
\hat{H}=-\frac{\hbar^{2}}{2} \nabla \cdot\left(\frac{1}{m(\lambda, x)} \nabla\right)+V(x)
$$

where $\hbar$ is the reduced Planck constant, and $\nabla$ denotes the spatial gradient.

Assuming non-parabolicity for the electron's dispersion relation, the electron effective mass $m(\lambda, x)$ is constant on $\Omega_{q}$ and on the matrix $\Omega_{m}$ for every fixed energy level $\lambda$, and is taken as $[1,3]$

$$
\frac{1}{m_{j}(\lambda)}:=\left.\frac{1}{m(\lambda, x)}\right|_{x \in \Omega_{j}}=\frac{P_{j}^{2}}{\hbar^{2}}\left(\frac{2}{\lambda+E_{g, j}-V_{j}}+\frac{1}{\lambda+E_{g, j}-V_{j}+\Delta_{j}}\right),
$$

for $j \in\{m, q\}$, where the confinement potential $V_{j}:=\left.V\right|_{\Omega_{j}}$ is piecewise constant, and $P_{j}, E_{g, j}$ and $\Delta_{j}$ are the momentum matrix element, the band gap, and the spin-orbit splitting in the valence band for the quantum dot material $(j=q)$ and the matrix $(j=m)$, respectively. 


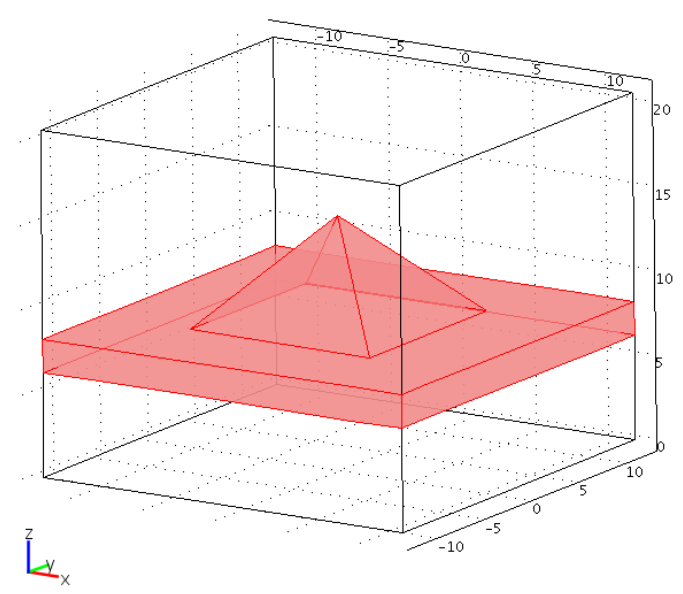

Fig. 1: Quantum dot with wetting layer

To determine the relevant energy states and corresponding wave functions $\psi$ we have to solve the governing Schrödinger equation

$$
-\nabla \cdot\left(\frac{\hbar^{2}}{2 m_{j}(\lambda)} \nabla \psi\right)+V(x) \psi=\lambda \psi, x \in \Omega_{q} \cup \Omega_{m}
$$

Since the wave function decays outside the quantum dot (and possibly the wetting layer) very rapidly, it is reasonable to assume homogeneous Dirichlet conditions $\psi=0$ on the horizontal part $\partial \Omega_{h}$ of the outer boundary of $\Omega_{m}$ (cf. Fig. 1). Following [12] we impose Neumann boundary conditions $\frac{\partial \psi}{\partial n}=0$ on the vertical part $\partial \Omega_{v}$ of the outer boundary of $\Omega_{m}$, since far away from the quantum dot wave functions must approach asymptotically ordinary quantum well envelope functions where the wetting layer is the quantum well. On the interface between the quantum dot material and the matrix the Ben Daniel-Duke condition [7] holds

$$
\left.\frac{1}{m_{q}} \frac{\partial \psi}{\partial n_{q}}\right|_{\partial \Omega_{q}}=\left.\frac{1}{m_{m}} \frac{\partial \psi}{\partial n_{m}}\right|_{\partial \Omega_{m}}, x \in \partial \Omega_{q} \cap \partial \Omega_{m}
$$

Here $n_{q}$ and $n_{m}$ denote the outward unit normal on the boundary of $\Omega_{q}$ and $\Omega_{m}$, respectively.

Let $\Omega:=\bar{\Omega}_{q} \cup \Omega_{m}$ and $H:=\left\{\psi \in H^{1}(\Omega): \psi=0\right.$ on $\left.\partial \Omega_{h}\right\}$. Multiplying equation (3) by $\phi \in H$ and integrating by parts, one gets the variational form 
of the Schrödinger equation

$$
\begin{aligned}
a(\psi, \phi ; \lambda):= & \frac{\hbar^{2}}{2 m_{q}(\lambda)} \int_{\Omega_{q}} \nabla \psi \cdot \nabla \phi d x+\frac{\hbar^{2}}{2 m_{m}(\lambda)} \int_{\Omega_{m}} \nabla \psi \cdot \nabla \phi d x+V_{q} \int_{\Omega_{q}} \psi \phi d x \\
& +V_{m} \int_{\Omega_{m}} \psi \phi d x=\lambda \int_{\Omega} \psi \phi d x=: \lambda b(\psi, \phi) \quad \text { for every } \phi \in H .
\end{aligned}
$$

In a similar way as in [20] it can be shown, that problem (5) has a countable set of positive eigenvalues $0<\lambda_{1} \leq \lambda_{2} \leq \cdots \rightarrow \infty$ of finite multiplicity which satisfy a minmax characterization. Namely, for fixed $\psi \neq 0$ the real equation

$$
f(\lambda ; \psi):=\lambda b(\psi, \psi)-a(\psi, \psi ; \lambda)=0
$$

has a unique positive solution $p(\psi)$. Hence, equation (6) defines a functional $p: H \rightarrow \mathbb{R}$ called Rayleigh functional (which generalizes the Rayleigh quotient for linear eigenproblems), and the $k$ :th smallest eigenvalue of (5) satisfies

$$
\lambda_{k}=\min _{\operatorname{dim} V=k} \max _{u \in V, u \neq 0} p(u) .
$$

Moreover, an eigenvalue $\tilde{\lambda}$ of (5) is the $k$ :th smallest eigenvalue if and only if $\mu=0$ is the $k$ :th largest eigenvalue of the linear eigenvalue problem

$$
\tilde{\lambda} b(\psi, \phi)-a(\psi, \phi ; \tilde{\lambda})=\mu b(\psi, \phi) \quad \text { for every } \phi \in H .
$$

Discretizing the Schrödinger equation (3) with the boundary and interface conditions specified above by a Galerkin method (finite elements, e.g.) one gets a rational matrix eigenvalue problem

$$
S(\lambda) x:=\lambda M x-\frac{1}{m_{q}(\lambda)} A_{q} x-\frac{1}{m_{m}(\lambda)} A_{m} x-B x=0
$$

where

$$
\begin{gathered}
A_{j}=\left(\int_{\Omega_{j}} \nabla \phi_{k} \cdot \nabla \phi_{\ell} d x\right)_{k, \ell}, j \in\{q, m\} \\
M=\left(\int_{\Omega} \phi_{k} \phi_{\ell} d x\right)_{k, \ell} \text { and } B=\left(V_{q} \int_{\Omega_{q}} \phi_{k} \phi_{\ell} d x+V_{m} \int_{\Omega_{m}} \phi_{k} \phi_{\ell} d x\right)_{k, \ell}
\end{gathered}
$$

and $\phi_{i}$ denotes a basis of the ansatz space.

$A_{q}, A_{m}$ and $B$ are symmetric and positive semi-definite, and $M$ is positive definite, and for $\lambda \geq 0$ the matrix

$$
\frac{\hbar^{2}}{2 m_{q}(\lambda)} A_{q}+\frac{\hbar^{2}}{2 m_{q}(\lambda)} A_{q}
$$

is positive definite. Hence, the eigenvalues of the dicretized problem (9) satisfy a minmax principle as well, and it follows from the minmax characterization (7) of the nonlinear Schrödinger equation that the $k$ :th smallest eigenvalue of the discretized problem (9) is an upper bound of the corresponding eigenvalue of problem (3). 


\section{Solving the discretized problem}

In this section we consider the problem to compute a few eigenvalues and corresponding eigenvectors at the lower end of the spectrum of the discretization (9) of the Schrödinger equation (3).

For linear sparse eigenproblems $S(\lambda)=\lambda B-A$ very efficient methods are iterative projection methods like the Lanczos, the Arnoldi, and the Jacobi-Davidson method, e.g., where approximations to the wanted eigenvalues and eigenvectors are obtained from projections of the eigenproblem to subspaces of small dimension which are expanded in the course of the algorithm.

Let $V \in \mathbb{R}^{n \times k}$ be an (orthonormal) basis of the current search space $\mathcal{V} \subset \mathbb{R}^{n}$, and assume that $\theta$ is an eigenvalue of the projected eigenvalue problem

$$
V^{T} S(\lambda) V y=0
$$

$y \in \mathbb{R}^{k}$ is a corresponding eigenvector, and denote by $x:=V y$ the corresponding Ritz vector. To obtain an improved approximation it is reasonable to expand $\mathcal{V}$ by a direction with a high approximation potential for the eigenvector wanted next.

There are two approaches in the literature for expanding the search space, both approximating inverse iteration: a Jacobi-Davidson type method [2] and the Arnoldi method [18] based on the residual inverse iteration. Here we restrict ourselves to the latter one.

Residual inverse iteration (introduced by Neumaier [16]) suggests the expansion

$$
v=S(\sigma)^{-1} S(\theta) x
$$

of the search space $\mathcal{V}$, where $\sigma$ is a fixed parameter close to the wanted eigenvalues.

For a linear eigenproblem $S(\lambda)=A-\lambda B$ this is exactly the Cayley transform with pole $\sigma$ and zero $\theta$, and since $(A-\sigma B)^{-1}(A-\theta B)=I+(\sigma-\theta)(A-\sigma B)^{-1} B$ and Krylov spaces are shift-invariant the resulting projection method expanding $\mathcal{V}$ by $v$ is nothing else but the shift-and-invert Arnoldi method.

If the linear system $S(\sigma) v=S(\theta) x$ is too expensive to solve for $v$ we may choose as new direction $v=K^{-1} S(\theta) x$ with $K \approx S(\sigma)$, and for the linear problem we obtain an inexact Cayley transform or a preconditioned Arnoldi method. The resulting iterative projection method given in Algorithm 1 therefore is called nonlinear Arnoldi method, although no Krylov space is constructed and no Arnoldi recursion holds.

There are many details that have to be considered when implementing the nonlinear Arnoldi method concerning the choice of the initial basis, when and how to update the preconditioner, and how to restart the method. A detailed discussion is given in [18].

A crucial point in iterative projection methods for general nonlinear eigenvalue problems when approximating more than one eigenvalue is to inhibit the method from converging to the same eigenvalue repeatedly. For linear eigenvalue problems this is easy to do by using Schur forms or generalized Schur forms for 


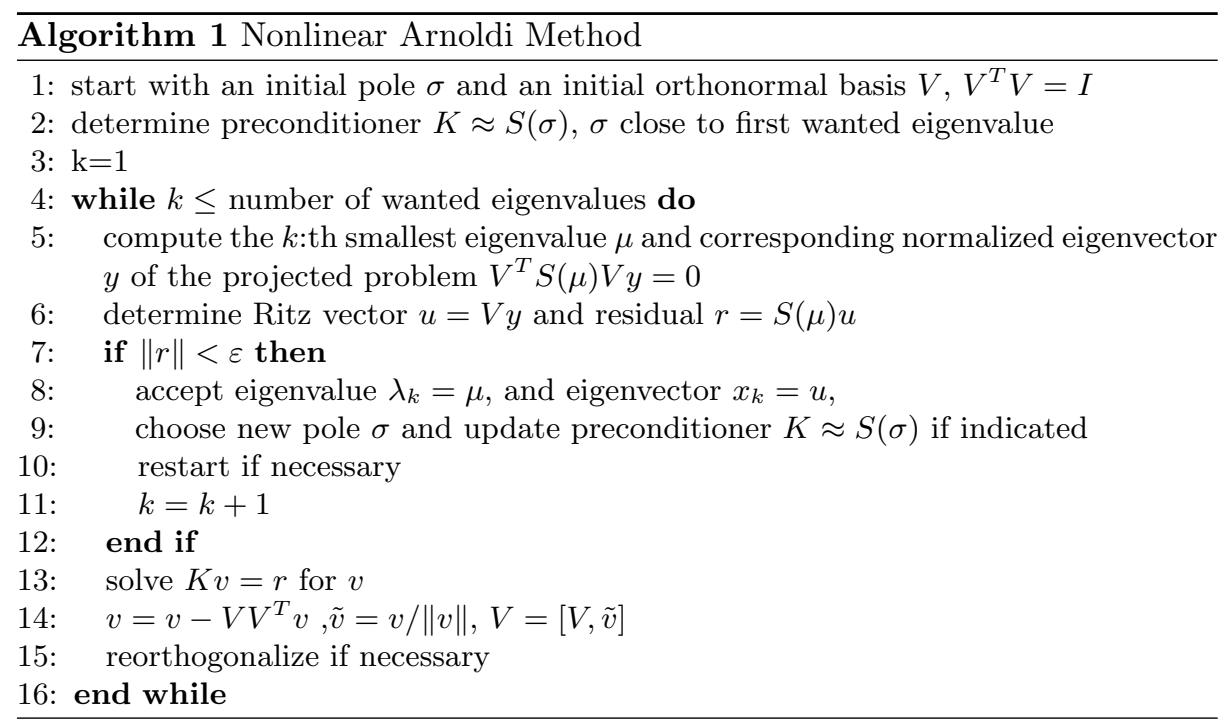

the projected problem and then locking or purging certain eigenvectors. For nonlinear problems, however, such Schur forms do not exist and this presents one of the most difficult tasks in achieving good convergence.

For symmetric nonlinear eigenproblems satisfying a minmax characterization however, its eigenvalues can be computed safely one after the other. The minimum in (7) is attained by the invariant subspace of $S\left(\lambda_{k}\right)$ corresponding to the $k$ :th largest eigenvalues, and the maximum by every eigenvector corresponding to the eigenvalue 0 . This suggests the safeguarded iteration for computing the $k$ :th smallest eigenvalue which reads as follows for the projected eigenproblem $P(\lambda) y:=V^{T} S(\lambda) V y=0$ :

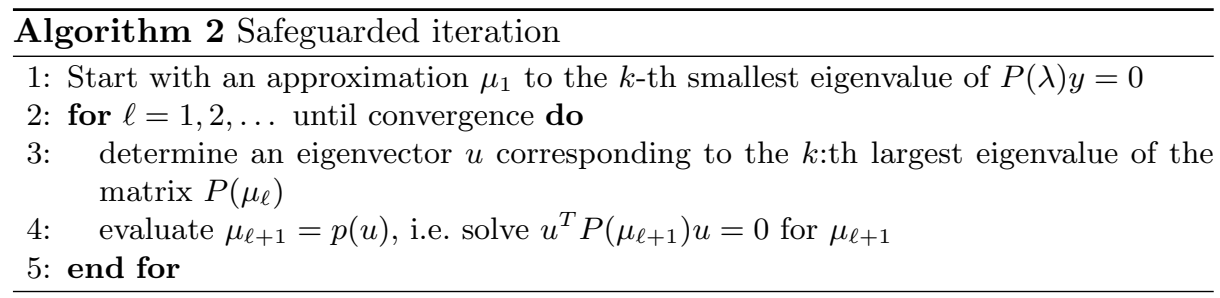

The safeguarded iteration has the following convergence properties [17]: It converges globally to the smallest eigenvalue $\lambda_{1}$. The (local) convergence to simple eigenvalues is quadratic. If $P^{\prime}(\lambda)$ is positive definite, and $u$ in Step 3 of the last algorithm is replaced by an eigenvector of $P\left(\mu_{\ell}\right) u=\mu P^{\prime}\left(\mu_{\ell}\right) u$ corresponding to the $k$ :th largest eigenvalue, then the convergence is even cubic. Moreover, a variant exists which is globally convergent also for higher eigenvalues. 


\section{Numerical experiments}

We consider a pyramidal quantum dot with width $12.4 \mathrm{~nm}$ and height $6.2 \mathrm{~nm}$ embedded in a cuboid matrix of size $24.8 \mathrm{~nm} \times 24.8 \mathrm{~nm} \times 18.6 \mathrm{~nm}$. We computed the band structure for the pure dot without a wetting layer, for the combined quantum dot and wetting layer structures (cf. Fig. 1) for two wetting layers of thickness $1 \mathrm{~nm}$ and $2 \mathrm{~nm}$, respectively, and for the pure wetting layer.

In the calculations of the electron energy spectra for a narrow gap InAs quantum dot in a GaAs matrix we used the semiconductor band structure parameters for InAs as: $P_{q}=0.8503, g_{q}=0.42, \delta_{q}=0.48$, and $V_{q}=0$, and for GaAs we chose $P_{m}=0.8878, g_{m}=1.52, \delta_{m}=0.34$, and $V_{m}=0.77$ as in [9].

Using FEMLAB [4] we discretized the Schrödinger equation by the finite element method with quadratic Lagrangian elements on a tetrahedral grid. Since the envelope functions are mainly concentrated on the quantum dot (which occupies only less than $3 \%$ of $\Omega$ ) and the wetting layer, and since they decay very rapidly outside the quantum dot/wetting layer structure, we chose a nonuniform grid such that roughly half of the degrees of freedom are in the InAs structure and the remaining ones are in the GaAs matrix.

The arising rational eigenvalue problems were solved under MATLAB 7.0.4 on an Intel Pentium D processor with 4 GByte RAM and $3.2 \mathrm{GHz}$ by the nonlinear Arnoldi method, where the projected eigenproblems were solved by the safeguarded iteration. We started the method with a constant vector on $\bar{\Omega}_{q} \cup \Omega_{m}$ which is far away from an eigenvector, and we terminated the iteration for an eigenvalue, if the residual norm was less than $10^{-8}$. Due to the symmetry of the problem there exist multiple eigenvalues (for instance the second eigenvalue in all cases). The Arnoldi method had no problems to detect these multiple eigenvalues with the right multiplicity.

We first consider the pure quantum dot problem which has five energy eigenvalues smaller than the confinement potential $V_{m}=0.77$ displayed in the second column of Tab. 1. The discretized problem has 183124 degrees of freedom, and it takes 297.8 seconds to solve it. The envelope functions $\psi_{j}$ corresponding to these states are essentially confined to the quantum dot. Fig. $2-4$. show on the left a cut $\left\{\psi_{j}(0, y, z):(0, y, z) \in \bar{\Omega}\right\}$ through $\psi_{j}$ for $j=1,2,4 . \psi_{3}$ is obtained from $\psi_{2}$ rotating it about the $z$-axis by 90 degrees, and $\psi_{5}$ is skew-symmetric with respect to the plain $\{0, y, z): y, z \in \mathbb{R}\}$ (and therefore its cut is identical to 0$)$.

Next we added to the quantum dot a wetting layer of thickness $1 \mathrm{~nm}$ and $2 \mathrm{~nm}$, respectively. In this case there are 18 and 31 eigenvalues smaller than $V_{m}$, respectively, most of them being approximate quantum well eigenstates corresponding to pure the wetting layer. The smallest 5 eigenvalues are shown in columns 3 and 4 in Tab. 1 . They are substantially smaller than the corresponding ones of the pure quantum dot. For instance, the ground state is smaller by 14 and 26 percent, respectively.

The envelope functions $\psi_{1}, \psi_{2}$ and $\psi_{4}$ for the case of a wetting layer of thickness $1 \mathrm{~nm}$ are displayed in Fig. 2 - 4. on the right. While the ground state is localized quite well to the quantum dot, this property gets lost for excited 
eigenstates. For the wetting layer of thickness $2 \mathrm{~nm}$ this is even more pronounced. The envelope functions $\psi_{1}$ and $\psi_{2}$ for this case are shown in Fig. 5.

Table 1. Electronic eigenstates

\begin{tabular}{|r|r|r|r|r|}
\hline & pure QD & QD/WL 1 nm & QD/WL 2nm & pure WL 2 nm \\
\hline dimension & 183124 & 156479 & 152928 & 10903 \\
\hline CPU & 297.8 & 251.2 & 235.0 & 46.4 \\
\hline$\lambda_{1}$ & 0.41621 & 0.35741 & 0.30864 & 0.45720 \\
$\lambda_{2 / 3}$ & 0.59909 & 0.51472 & 0.43239 & 0.47149 \\
$\lambda_{4}$ & 0.71802 & 0.60738 & 0.46590 & 0.48545 \\
$\lambda_{5}$ & 0.72956 & 0.61991 & 0.47964 & 0.51246 \\
\hline
\end{tabular}

$u(0, y, z): \quad \lambda(1)=0.416211$

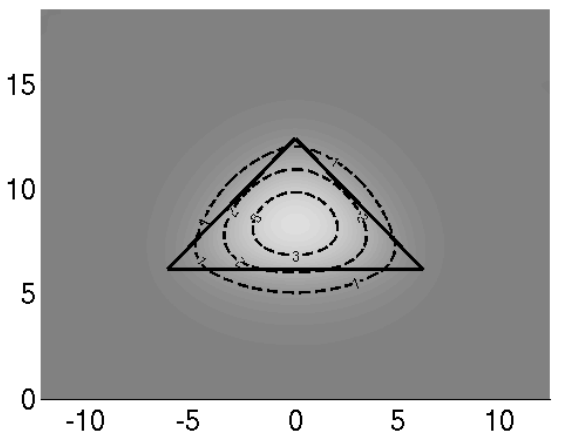

$u(0, y, z): \quad \lambda(1)=0.357406$

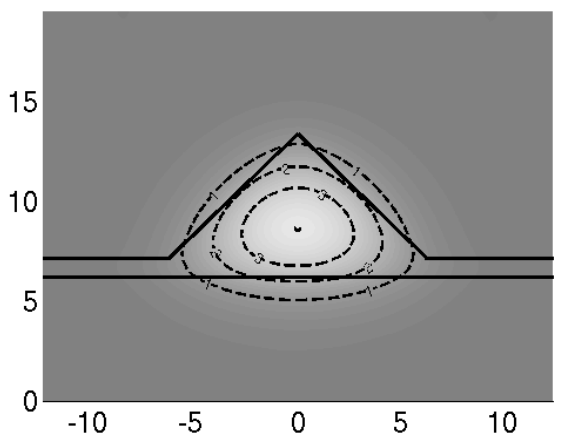

Fig. 2: 1. eigenvector of quantum dot without and with wetting layer

\section{References}

1. G. Bastard. Wave Mechanics Applied to Semiconductor Heterostructures. Les editions de physique, Les Ulis Cedex, 1988.

2. T. Betcke and H. Voss. A Jacobi-Davidson-type projection method for nonlinear eigenvalue problems. Future Generation Computer Systems, 20(3):363 - 372, 2004.

3. S.L. Chuang. Physics of Optoelectronic Devices. John Wiley \& Sons, New York, 1995.

4. FEMLAB, Version 3.1. COMSOL, Inc., Burlington, MA, USA, 2004.

5. I. Filikhin, E. Deyneka, G. Melikian, and B. Vlahovic. Electron states of semiconductor quantum ring with geometry and size variations. Molecular Simulation, $31: 779-785,2005$.

6. I. Filikhin, E. Deyneka, and B. Vlahovic. Energy dependent effective mass model of InAs/GaAs quantum ring. Model.Simul.Mater.Sci.Eng., 12:1121 - 1130, 2004. 

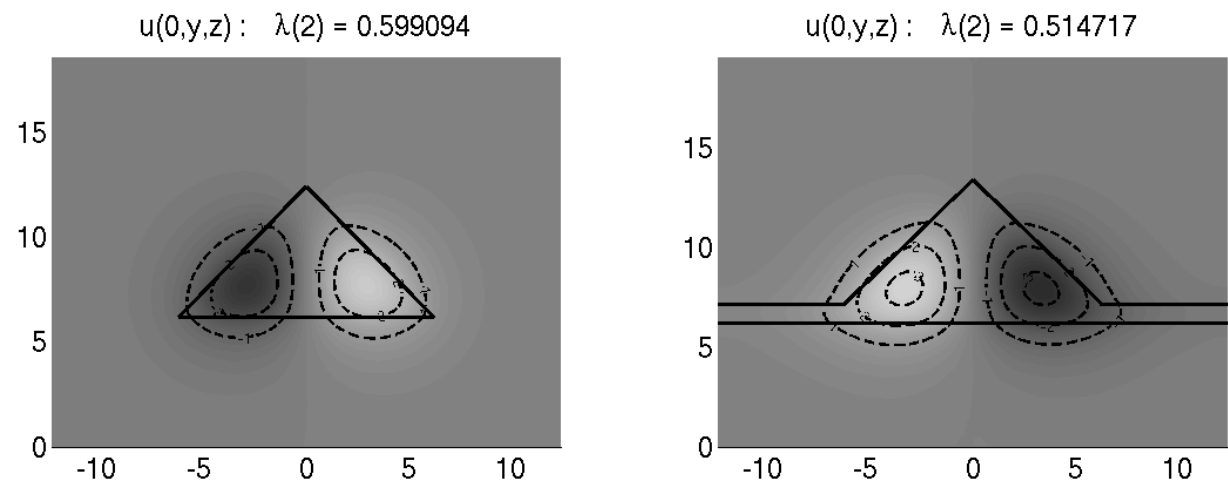

Fig. 3: 2. eigenvector of quantum dot without and with wetting layer
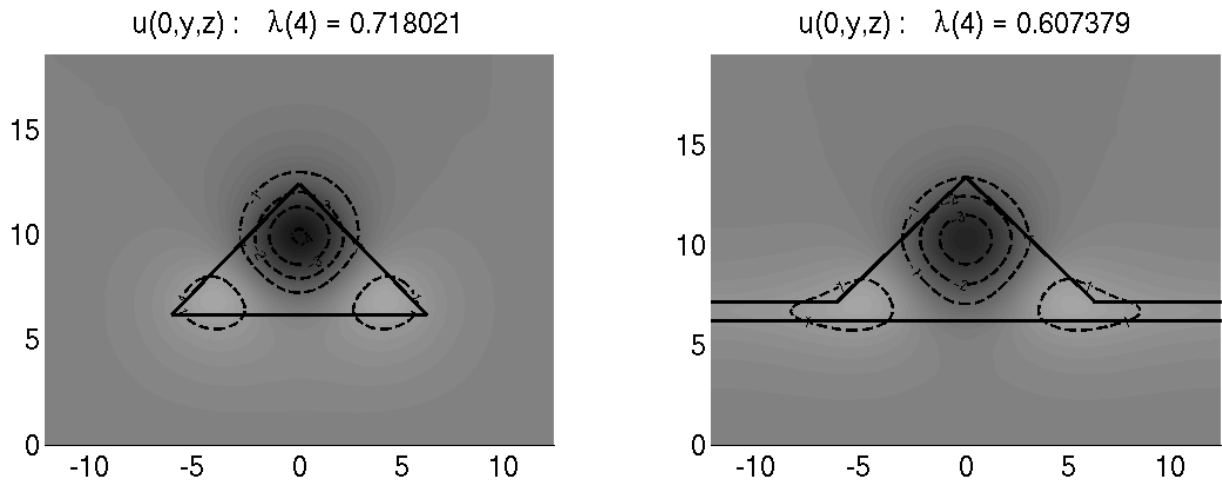

Fig. 4: 4. eigenvector of quantum dot without and with wetting layer

7. P. Harrison. Quantum Wells, Wires and Dots. Theoretical and Computational Physics. John Wiley \& Sons, Chicester, 2000.

8. T.-M. Hwang, W.-W. Lin, J.-L. Liu, and W. Wang. Jacobi-Davidson methods for cubic eigenvalue problems. Numer.Lin.Alg.Appl., 12:605 - 624, 2005.

9. T.-M. Hwang, W.-W. Lin, W.-C. Wang, and W. Wang. Numerical simulation of three dimensional quantum dot. J. Comput.Phys., 196:208 - 232, 2004.

10. Y. Li. Numerical calculation of electronic structure for three-dimensional nanoscale semiconductor quantum dots and rings. J. Comput. Electronics, 2:49 - 57, 2003.

11. Y. Li, O. Voskoboynikov, C.P. Lee, and S.M. Sze. Computer simulation of electron energy level for different shape InAs/GaAs semiconductor quantum dots. Comput.Phys.Comm., 141:66 - 72, 2001.

12. R.V. Melnik and M. Willatzen. Modelling coupled motion of electrons in quantum dots with wetting layers. In Proceedings of the 5th Internat. Conference on Modelling and Simulation of Microsystems, MSM 2002, pages 506 - 509, Puerto Rico, USA, 2002.

13. R.V. Melnik and M. Willatzen. Bandstructures of conical quantum dots with wetting layers. Nanotechnology, 15:1 - 8, 2004.

14. R.V. Melnik and K.N. Zotsenko. Computations of coupled electronic states in quantum dot/wetting layer cylindrical structures. In P.M.A. Sloot, D. Abramson, 

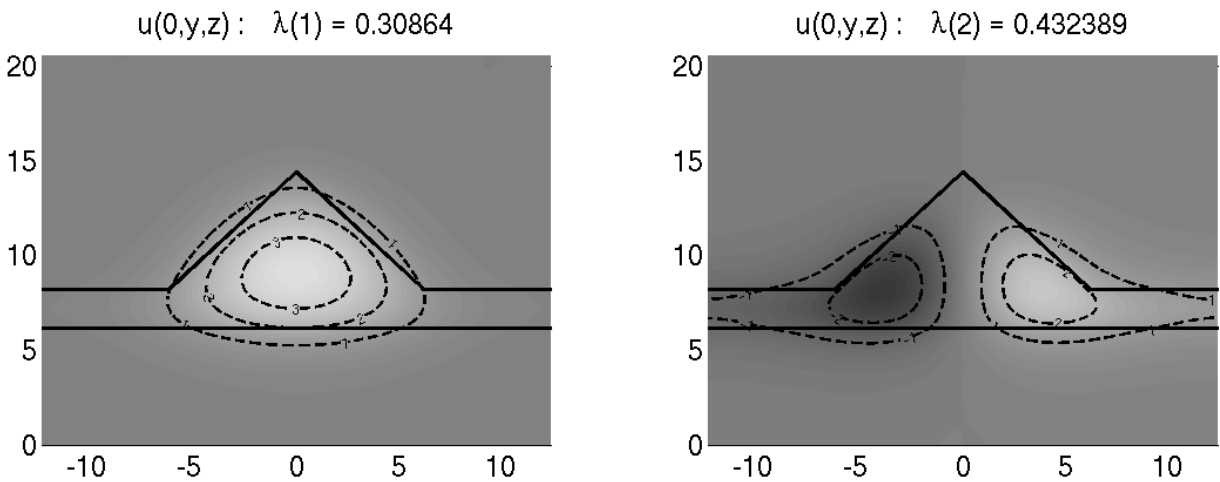

Fig. 5: 1. and 2. eigenvector of quantum dot with thick wetting layer

A.V. Bogdanov, J.J. Dongarra, A.Y. Zomaya, and Y.E. Gorbatchev, editors, Computational Science - ICCS 2002, 3rd International Conference,Proceedings, Part III, volume 2659 of Lecture Notes in Computer Science, pages 343-349, Berlin, 2003. Springer Verlag.

15. R.V. Melnik and K.N. Zotsenko. Finite element analysis of coupled electronic states in quantum dot nanostructures. Modelling Simul. Mater. Sci. Eng., 12:465 - 477, 2004.

16. A. Neumaier. Residual inverse iteration for the nonlinear eigenvalue problem. SIAM J. Numer. Anal., 22:914 - 923, 1985.

17. H. Voss. Initializing iterative projection methods for rational symmetric eigenproblems. In Online Proceedings of the Dagstuhl Seminar Theoretical and Computational Aspects of Matrix Algorithms, Schloss Dagstuhl 2003, ftp://ftp.dagstuhl.de/pub/Proceedings/03/03421/03421.VoszHeinrich.Other.pdf, 2003.

18. H. Voss. An Arnoldi method for nonlinear eigenvalue problems. BIT Numerical Mathematics, 44:387 - 401, 2004.

19. H. Voss. Electron energy level calculation for quantum dots. Technical Report 91, Institute of Numerical Simulation, Hamburg University of Technology, 2005. To appear in Comput. Phys. Comm.

20. H. Voss. A rational eigenvalue problem governing relevant energy states of a quantum dots. Technical Report 92, Institute of Numerical Simulation, Hamburg University of Technology, 2005. Submitted to J. Comput. Phys. 